\title{
Product of differentiation and composition operators on the logarithmic Bloch space
}

\author{
Jizhen Zhou ${ }^{1}$ and Xiangling Zhu ${ }^{2 *}$
}

"Correspondence: jyuzx|@163.com ${ }^{2}$ Faculty of Information Technology, Macau University of Science and Technology, Avenida Wai Long, Taipa, Macau, China Full list of author information is available at the end of the article

\section{Abstract}

We obtain a criterion for the boundedness and compactness of the products of differentiation and composition operators $C_{\varphi} D^{m}$ on the logarithmic Bloch space in terms of the sequence $\left\{z^{n}\right\}$. An estimate for the essential norm of $C_{\varphi} D^{m}$ is given. MSC: $47 \mathrm{~B} 38 ; 30 \mathrm{H} 30$

\section{Introduction}

Denote by $H(\mathbb{D})$ the space of all analytic functions on the unit disk $\mathbb{D}=\{z:|z|<1\}$ in the complex plane. Let $H^{\infty}=H^{\infty}(\mathbb{D})$ denote the space of bounded analytic functions on $\mathbb{D}$. An $f \in H(\mathbb{D})$ is said to belong to the Bloch space $\mathcal{B}$ if

$$
\|f\|_{\mathcal{B}}=\sup _{z \in \mathbb{D}}\left|f^{\prime}(z)\right|\left(1-|z|^{2}\right)<\infty
$$

The logarithmic-Bloch space, denoted by $\mathcal{L B}$, consists of all $f \in H(\mathbb{D})$ satisfying

$$
\|f\|_{\log }=\sup _{z \in \mathbb{D}}(1-|z|)\left|f^{\prime}(z)\right| \log \frac{e}{1-|z|}<\infty .
$$

$\mathcal{L B}$ is a Banach space with the norm $\|f\|_{\mathcal{L B}}=|f(0)|+\|f\|_{\text {log }}$. It is well known that $\mathcal{L B} \cap H^{\infty}$ is the space of multipliers of the Bloch space $\mathcal{B}$ (see $[1,2]$ ). For some results on logarithmictype spaces and operators on them, see, for example, [3-10].

Let $\varphi$ be an analytic self-map of $\mathbb{D}$. The composition operator $C_{\varphi}$ is defined by

$$
C_{\varphi}(f)=f \circ \varphi, \quad f \in H(\mathbb{D}) .
$$

The differentiation operator $D$ is defined by $D f=f^{\prime}, f \in H(\mathbb{D})$. For a nonnegative integer $m \in \mathbb{N}$, we define

$$
D^{m} f=f^{(m)}, \quad f \in H(\mathbb{D}) .
$$

The product of differentiation and composition operators $C_{\varphi} D^{m}$ is defined as follows:

$$
C_{\varphi} D^{m} f=f^{(m)} \circ \varphi, \quad f \in H(\mathbb{D}) .
$$


A basic problem concerning concrete operators on various Banach spaces is to relate the operator theoretic properties of the operators to the function theoretic properties of their symbols, which attracted a lot of attention recently, the reader can refer to [4-37].

It is a well-known consequence of the Schwarz-Pick lemma that the composition operator is bounded on $\mathcal{B}$. See $[21-24,27,33-35,37]$ for the study of composition operators and weighted composition operators on the Bloch space. The product-type operators on or into Bloch type spaces have been studied in many papers recently; see [12-20, 26, 28 32, 34, 36] for example.

Let $X$ and $Y$ be two Banach spaces. Recall that a linear operator $T: X \rightarrow Y$ is said to be compact if it takes bounded sets in $X$ to sets in $Y$ which have compact closure. The essential norm of an operator $T$ between $X$ and $Y$ is the distance to the compact operators $K$, that is, $\|T\|_{e}^{X \rightarrow Y}=\inf \{\|T-K\|: K$ is compact $\}$, where $\|\cdot\|$ is the operator norm. It is easy to see that $\|T\|_{e}^{X \rightarrow Y}=0$ if and only if $T$ is compact. For some results in the topic, see, for example, [11, 20, 22, 24, 26, 28, 37].

In [34], Wu and Wulan obtained a characterization for the compactness of the product of differentiation and composition operators acting on the Bloch space as follows:

Theorem A Let $\varphi$ be an analytic self-map of $\mathbb{D}, m \in \mathbb{N}$. Then $C_{\varphi} D^{m}: \mathcal{B} \rightarrow \mathcal{B}$ is compact if and only if

$$
\lim _{n \rightarrow \infty}\left\|C_{\varphi} D^{m}\left(z^{n}\right)\right\|_{\mathcal{B}}=0
$$

The purpose of the paper is to extend Theorem A to the case of $\mathcal{L B}$. We will characterize the boundedness and compactness of $C_{\varphi} D^{m}$ in terms of the sequence $\left\{z^{n}\right\}$. Moreover, an estimate for the essential norm of $C_{\varphi} D^{m}$ will be given. The main results are given in Sections 3 and 4 .

In the paper, we say that a real sequence $\left\{a_{n}\right\}_{n \in \mathbb{N}}$ is asymptotic to another real sequence of $\left\{b_{n}\right\}_{n \in \mathbb{N}}$ and write ' $a_{n} \sim b_{n}$ ' if and only if

$$
\lim _{n \rightarrow \infty} \frac{a_{n}}{b_{n}}=1 .
$$

In addition, we say that $A \preceq B$ if there exists a constant $C$ such that $A \leq C B$. The symbol $A \approx B$ means that $A \preceq B \preceq A$.

\section{Auxiliary lemmas}

In this section, we state and prove some auxiliary results which will be used to prove the main results in this paper.

Lemma 2.1 For $m, n \in \mathbb{N}$, define the function $H_{m, n}:[0,1) \rightarrow[0, \infty)$ by

$$
H_{m, n}(x)=\frac{n !}{(n-m-1) !} x^{n-m-1}(1-x)^{m+1} \log \frac{e}{1-x} .
$$

Then the following statements hold:

(i) For $n, m \in \mathbb{N}$ and $n \geq m+1$, there is a unique $x_{m, n} \in[0,1)$ such that $H_{m, n}\left(x_{m, n}\right)$ is the absolute maximum of $H_{m, n}$. 
(ii)

$$
\lim _{n \rightarrow \infty} x_{m, n}=1
$$

and

$$
\lim _{n \rightarrow \infty}\left[n\left(1-x_{m, n}\right)\right]=m+1
$$

(iii)

$$
\lim _{n \rightarrow \infty} \frac{\max _{0<t<1} H_{m, n}(t)}{\log (n+1)}=\left(\frac{m+1}{e}\right)^{m+1}
$$

Proof Directly computing we have

$$
H_{m, n}^{\prime}(x)=\frac{n !}{(n-m-1) !} x^{n-m-2}(1-x)^{m}\left((n-m-1-n x) \log \frac{e}{1-x}+x\right) .
$$

Define

$$
g_{m, n}(x)=(n-m-1-n x) \log \frac{e}{1-x}+x, \quad x \in[0,1)
$$

It is easy to see that $g_{m, n}$ is continuous on $[0,1)$ and $g_{m, n}(0)=n-m-1 \geq 0, \lim _{x \rightarrow 1^{-}} g_{m, n}(x)=$ $-\infty$. Furthermore,

$$
g_{m, n}^{\prime}(x)=-n \log \frac{e}{1-x}+n-\frac{m+1}{1-x}+1<0, \quad x \in[0,1)
$$

Then $g_{m, n}$ is decreasing on $[0,1)$. When $n=m+1$, we get $\max _{0 \leq x<1} H_{m, n}(x)=H_{m, n}(0)$. When $n>m+1$, the intermediate value theorem of continuous function gives the result that there exists a unique $x_{m, n} \in(0,1)$ such that $g_{m, n}\left(x_{m, n}\right)=0$. So we have

$$
\max _{0<t<1} H_{m, n}(x)=H_{m, n}\left(x_{m, n}\right)
$$

(i) has been proved. By (2.5), we have $g_{m, n}\left(x_{m, n}\right)=0$. Thus

$$
\left(\frac{n-m-1}{n}-x_{m, n}\right) \log \frac{e}{1-x_{m, n}}=-\frac{x_{m, n}}{n} .
$$

It follows from $\lim _{n \rightarrow \infty} \frac{x_{m, n}}{n}=0$ and $\log \frac{e}{1-x_{m, n}} \geq 1$ that (2.2) holds. Also, $g_{m, n}\left(x_{m, n}\right)=0$ gives the result that

$$
\frac{n-m-1}{n}-x_{m, n}=-\frac{x_{m, n}}{n \log \frac{e}{1-x_{m, n}}} .
$$

So we have

$$
n\left(1-x_{m, n}\right)-m-1=-\frac{x_{m, n}}{\log \frac{e}{1-x_{m, n}}} .
$$

This gives the result (2.3). The proof of (ii) is complete. 
Note that

$$
n \log x_{m, n} \sim n \log \left[1+\left(x_{m, n}-1\right)\right] \sim n\left(x_{m, n}-1\right) \rightarrow-m-1 \quad \text { as } n \rightarrow \infty .
$$

This and (2.2) give

$$
\lim _{n \rightarrow \infty} x_{m, n}^{n-m-1}=e^{-m-1}
$$

By (2.3) and (2.6) we obtain

$$
\begin{aligned}
\lim _{n \rightarrow \infty} \frac{H_{m, n}\left(x_{m, n}\right)}{\log (n+1)} & =\lim _{n \rightarrow \infty} \frac{n ! x_{m, n}^{n-m-1}\left(1-x_{m, n}\right)^{m+1} \log \frac{e}{1-x_{m, n}}}{(n-m-1) ! \log (n+1)} \\
& =e^{-m-1} \lim _{n \rightarrow \infty} \frac{n !((m+1) / n)^{m+1} \log \frac{e n}{m+1}}{(n-m-1) ! \log (n+1)}=\left(\frac{m+1}{e}\right)^{m+1}
\end{aligned}
$$

which shows that (iii) hold. The proof is complete.

Lemma 2.2 Let $m, n \in \mathbb{N}$ and $n-m-1>0$. Let $r_{m, n}=(n-m-1) / n$. Then $H_{m, n}$ is increasing on $\left[r_{m, n-m}, r_{m, n}\right]$ and

$$
\min _{r_{m, n-m} \leq x \leq r_{m, n}} H_{m, n}(x)=H_{m, n}\left(r_{m, n-m}\right) \sim\left(\frac{m+1}{e}\right)^{m+1} \log (n+1) \quad \text { as } n \rightarrow \infty .
$$

Consequently,

$$
\min _{r_{m, n-m} \leq x \leq r_{m, n}} \frac{H_{m, n}(x)}{\left\|z^{n}\right\|_{\mathcal{L B}}}=\frac{H_{m, n}\left(r_{m, n-m}\right)}{\left\|z^{n}\right\|_{\mathcal{L B}}} \sim \frac{(m+1)^{m+1}}{e^{m}} \text { as } n \rightarrow \infty .
$$

Proof Since $n-m-1>0$, we have

$$
H_{m, n}^{\prime}\left(r_{m, n}\right)=\frac{n !}{(n-m-1) !}\left(\frac{n-m-1}{n}\right)^{n-m-2}\left(\frac{m+1}{n}\right)^{m}\left(\frac{n-m-1}{n}\right)>0 .
$$

By Lemma 2.1, we have $r_{m, n}<x_{m, n}$, where $x_{m, n}$ is given as in Lemma 2.1. Since $H_{m, n}^{\prime}(x)>0$ for $x \in\left(0, x_{m, n}\right)$, we see that $H_{m, n}$ is increasing on $\left[r_{m, n-m}, r_{m, n}\right]$. Thus

$$
\begin{aligned}
\min _{r_{m, n-m \leq x \leq r_{m, n}}} H_{m, n}(x) & =H_{m, n}\left(r_{m, n-m}\right) \\
& =\frac{n !}{(n-m-1) !}\left(\frac{n-2 m-1}{n-m}\right)^{n-m-1}\left(\frac{m+1}{n-m}\right)^{m+1} \log \frac{e(n-m)}{m+1} .
\end{aligned}
$$

Applying the important limit $\lim _{n \rightarrow \infty}\left(1+\frac{1}{n}\right)^{n}=e$ we obtain the result that (2.7) holds.

By Lemma 2.1 we have

$$
\left\|z^{n}\right\|_{\mathcal{L B}}=\sup _{|z|<1} n|z|^{n-1}(1-|z|) \log \frac{e}{1-|z|}=H_{0, n}\left(x_{0, n}\right),
$$


where $x_{0, n}$ is given in Lemma 2.1. By Lemma 2.1 we have

$$
\begin{aligned}
\lim _{n \rightarrow \infty} \frac{H_{m, n}\left(r_{m, n-m}\right)}{\left\|z^{n}\right\|_{\mathcal{L B}}} & =\lim _{n \rightarrow \infty} \frac{H_{m, n}\left(r_{m, n-m}\right)}{\log (n+1)} \frac{\log (n+1)}{\left\|z^{n}\right\|_{\mathcal{L B}}} \\
& =\lim _{n \rightarrow \infty} \frac{H_{m, n}\left(r_{m, n-m}\right)}{\log (n+1)} \lim _{n \rightarrow \infty} \frac{\log (n+1)}{\left\|z^{n}\right\|_{\mathcal{L B}}}=\frac{(m+1)^{m+1}}{e^{m}} .
\end{aligned}
$$

This gives (2.8). The proof is complete.

Lemma 2.3 [3] For $m \in \mathbb{N}$. Then $f \in \mathcal{L B}$ if and only if

$$
\sup _{z \in \mathbb{D}}(1-|z|)^{m}\left|f^{(m)}(z)\right| \log \frac{e}{1-|z|}<\infty .
$$

Moreover,

$$
\|f\|_{\mathcal{L B}} \approx \sum_{j=0}^{m-1}\left|f^{(j)}(0)\right|+\sup _{z \in \mathbb{D}}(1-|z|)^{m}\left|f^{(m)}(z)\right| \log \frac{e}{1-|z|} .
$$

\section{The boundedness of $C_{\varphi} D^{m}$ on $\mathcal{L B}$}

In this section, we will state the boundedness criterion for the operator $C_{\varphi} D^{m}$ on $\mathcal{L B}$. Since the boundedness of $C_{\varphi} D^{m}$ on $\mathcal{L B}$ gives $\varphi \in \mathcal{L B}$, we may always assume that $\varphi \in \mathcal{L B}$. The main result of this section is stated as follows.

Theorem 3.1 Let $m \in \mathbb{N}$ and $\varphi$ be an analytic self-map of $\mathbb{D}$ such that $\varphi \in \mathcal{L B}$. Then $C_{\varphi} D^{m}$ is bounded on $\mathcal{L B}$ if and only if

$$
\sup _{n \in \mathbb{N}} \frac{\left\|C_{\varphi} D^{m}\left(z^{n}\right)\right\|_{\mathcal{L B}}}{\left\|z^{n}\right\|_{\mathcal{L B}}}<\infty
$$

Proof $\Rightarrow$ ) Assume that $C_{\varphi} D^{m}$ is bounded on $\mathcal{L B}$, that is, $\left\|C_{\varphi} D^{m}\right\|_{\mathcal{L B} \rightarrow \mathcal{L B}}<\infty$. Since the sequence $\left\{z^{n} /\left\|z^{n}\right\|_{\mathcal{L B}}\right\}$ is bounded in the logarithmic Bloch space $\mathcal{L B}$, we have

$$
\frac{\left\|C_{\varphi} D^{m}\left(z^{n}\right)\right\|_{\mathcal{L B}}}{\left\|z^{n}\right\|_{\mathcal{L B}}} \leq\left\|C_{\varphi} D^{m}\right\|_{\mathcal{L B} \rightarrow \mathcal{L B}}\left\|\frac{z^{n}}{\left\|z^{n}\right\|_{\mathcal{L B}}}\right\|_{\mathcal{L B}} \leq\left\|C_{\varphi} D^{m}\right\|_{\mathcal{L B} \rightarrow \mathcal{L B}}<\infty
$$

for any $n \in \mathbb{N}$, from which the implication follows.

$\Leftarrow)$ We now assume that the condition (3.1) holds. On the one hand, for the case $\sup _{z \in \mathbb{D}}|\varphi(z)|<1$, there is an $r \in(0,1)$ such that $|\varphi(z)|<r$. By (3.1), for any given $f \in \mathcal{L B}$, we have

$$
\begin{aligned}
\left\|C_{\varphi} D^{m} f\right\|_{\mathcal{L B}} & =\sup _{z \in \mathbb{D}}(1-|z|) \log \frac{e}{1-|z|}\left|f^{(m+1)}(\varphi(z)) \varphi^{\prime}(z)\right| \\
& \leq \sup _{z \in \mathbb{D}}\|\varphi\|_{\mathcal{L B}} \frac{\left|f^{(m+1)}(\varphi(z))\right|(1-|\varphi(z)|)^{m+1} \log \frac{e}{1-|\varphi(z)|}}{(1-|\varphi(z)|)^{m+1} \log \frac{e}{1-|\varphi(z)|}} \\
& \preceq \sup _{z \in \mathbb{D}} \frac{\|\varphi\|_{\mathcal{L B}}\|f\|_{\mathcal{L B}}}{(1-r)^{m+1} \ln \frac{e}{1-r}}<\infty .
\end{aligned}
$$

The last estimate shows that the operator $C_{\varphi}$ is bounded on $\mathcal{L B}$. 
On the other hand, for the case $\sup _{z \in \mathbb{D}}|\varphi(z)|=1$. Let $N$ be the smallest positive integer such that $\mathbb{D}_{N}$ is not empty, where

$$
\mathbb{D}_{n}=\left\{z \in \mathbb{D}: r_{m, n-m} \leq|\varphi(z)| \leq r_{m, n}\right\}
$$

and $r_{m, n}$ is given in Lemma 2.2. Note that $H_{m, n}(|\varphi(z)|)>0$, when $z \in \mathbb{D}_{n}, n \geq N$, by (2.8) we obtain

$$
\epsilon:=\inf _{z \in \mathbb{D}_{n}} \frac{H_{m, n}(|\varphi(z)|)}{\left\|z^{n}\right\|_{\mathcal{L B}}}>0
$$

For any given $f \in \mathcal{L B}$, by Lemma 2.3 we have

$$
\begin{aligned}
\left\|C_{\varphi} D^{m} f\right\|_{\mathcal{L B}} & =\sup _{z \in \mathbb{D}}(1-|z|) \log \frac{e}{1-|z|}\left|f^{(m+1)}(\varphi(z)) \varphi^{\prime}(z)\right| \\
& =\sup _{n \geq N} \sup _{z \in \mathbb{D}_{n}}(1-|z|) \log \frac{e}{1-|z|}\left|f^{(m+1)}(\varphi(z)) \varphi^{\prime}(z)\right| \\
& =\sup _{n \geq N} \sup _{z \in \mathbb{D}_{n}}(1-|z|) \log \frac{e}{1-|z|}\left|f^{(m+1)}(\varphi(z)) \varphi^{\prime}(z)\right| \frac{\left\|z^{n}\right\|_{\mathcal{L B}}}{H_{m, n}(|\varphi(z)|)} \frac{H_{m, n}(|\varphi(z)|)}{\left\|z^{n}\right\|_{\mathcal{L B}}} \\
& \leq \frac{\|f\|_{\mathcal{L B}}}{\epsilon} \sup _{n \geq N} \sup _{z \in \mathbb{D}_{n}} \frac{n !}{(n-m-1) !}(1-|z|) \log \frac{e}{1-|z|}\left|\varphi^{\prime}(z)\right| \frac{|\varphi(z)|^{n-m-1}}{\left\|z^{n}\right\|_{\mathcal{L B}}} \\
& \leq \frac{\|f\|_{\mathcal{L B}}}{\epsilon} \sup _{n \geq N} \frac{\left\|C_{\varphi} D^{m}\left(z^{n}\right)\right\|_{\mathcal{L B}}}{\left\|z^{n}\right\|_{\mathcal{L B}}} .
\end{aligned}
$$

The proof is complete.

\section{The essential norm of $C_{\varphi} D^{m}$ on $\mathcal{L B}$}

Denote $K_{r} f(z)=f(r z)$ for $r \in(0,1)$. Then $K_{r}$ is a compact operator on the space $\mathcal{L B}$. It is easy to see that $\left\|K_{r}\right\| \leq 1$. We denote by $I$ the identity operator.

In order to give the lower and upper estimate for the essential norm of $C_{\varphi} D^{m}$ on $\mathcal{L B}$, we need the following result.

Lemma 4.1 There is a sequence $\left\{r_{k}\right\}$, with $0<r_{k}<1$ tending to 1 , such that the compact operator

$$
L_{n}=\frac{1}{n} \sum_{k=1}^{n} K_{r_{k}}
$$

on $\mathcal{L B}$ satisfies:

(i) for any $t \in(0,1), \lim _{n \rightarrow \infty} \sup _{\|f\|_{\mathcal{L B} \leq t}} \sup _{|z| \leq t}\left|\left(\left(I-L_{n}\right) f\right)^{\prime}(z)\right|=0$,

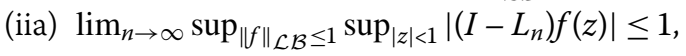

(iib) $\lim _{n \rightarrow \infty} \sup _{\|f\|_{\mathcal{L B}} \leq 1} \sup _{|z|<s}\left|\left(I-L_{n}\right) f(z)\right|=0$, for any $s \in(0,1)$,

(iii) $\lim \sup _{n \rightarrow \infty}\left\|I-L_{n}\right\| \leq 1$.

Proof (i) follows from (iib) by Cauchy's formula. The proof of (iii) is similar to the proof of Proposition 8 in [25]. Hence we omit it. Next we prove (iia) and (iib). The argument is much like that given in the proof of Proposition 2.1 of [25] or Lemmas 1 and 2 in [22]. For 
any $0<s<1$, we choose an increasing sequence $r_{k}$ tending to 1 such that $r_{k} \geq 1-\frac{1-s}{k^{2}}$. For any given $z \in \mathbb{D}$ and $r_{k}, k=1,2,3, \ldots$, there exists an $s_{k} \in\left(r_{k}, 1\right)$ such that

$$
\left|f(z)-f_{r_{k}}(z)\right|=z f^{\prime}\left(s_{k} z\right)\left(z-r_{k} z\right)
$$

For any $f \in \mathcal{L B}$ with $\|f\|_{\mathcal{L B}} \leq 1$, we have

$$
\begin{aligned}
\left|\left(I-L_{n}\right) f(z)\right| & \leq \frac{1}{n} \sum_{k=1}^{n}\left|f(z)-f_{r_{k}}(z)\right| \leq \frac{1}{n} \sum_{k=1}^{n}\left|f^{\prime}\left(s_{k} z\right)\right|\left(1-r_{k}\right) \\
& \leq \frac{1}{n} \sum_{k=1}^{n} \frac{1-r_{k}}{\left(1-\left|r_{k} z\right|\right) \log \frac{e}{1-\left|r_{k} z\right|}} \leq \frac{1}{n} \sum_{k=1}^{n} 1=1 .
\end{aligned}
$$

Thus

$$
\limsup _{n \rightarrow \infty} \sup _{\|f\|_{\mathcal{L} \mathcal{B}} \leq 1} \sup _{|z|<1}\left|\left(I-L_{n}\right) f(z)\right| \leq 1
$$

This shows that (iia) holds.

If $|z| \leq s$, by the equality (4.1), we have

$$
\begin{aligned}
\left|\left(I-L_{n}\right) f(z)\right| & \leq \frac{1}{n} \sum_{k=1}^{n} \frac{1-r_{k}}{(1-|s z|) \log \frac{e}{1-|s z|}} \\
& \leq \frac{1}{n} \sum_{k=1}^{n} \frac{1-r_{k}}{(1-s)}=\frac{1}{n} \sum_{k=1}^{n} \frac{1}{k^{2}} \leq \frac{\pi^{2}}{6 n} .
\end{aligned}
$$

The above estimate gives (iib). The proof is complete.

The following lemma can be proved in a standard way; see, for example Proposition 3.11 in [11].

Lemma 4.2 Let $m \in \mathbb{N}$ and $\varphi$ be an analytic self-map of $\mathbb{D}$. Then $C_{\varphi} D^{m}$ is compact on $\mathcal{L B}$ if and only if $C_{\varphi} D^{m}$ is bounded on $\mathcal{L B}$ and for any bounded sequence $\left\{f_{n}\right\}$ in $\mathcal{L B}$ which converges to zero uniformly on compact subsets of $\mathbb{D}$, then $\left\|C_{\varphi} D^{m} f_{n}\right\|_{\mathcal{L B}} \rightarrow 0$ as $n \rightarrow \infty$.

Theorem 4.3 Let $m \in \mathbb{N}$ and $\varphi$ be an analytic self-map of $\mathbb{D}$. Suppose that $C_{\varphi} D^{m}$ is bounded on $\mathcal{L B}$. Then the estimate for the essential norm of $C_{\varphi} D^{m}$ on $\mathcal{L B}$ is

$$
\left\|C_{\varphi} D^{m}\right\|_{e}^{\mathcal{L B} \rightarrow \mathcal{L B}} \approx \limsup _{n \rightarrow \infty} \frac{\left\|C_{\varphi} D^{m}\left(z^{n}\right)\right\|_{\mathcal{L B}}}{\left\|z^{n}\right\|_{\mathcal{L B}}}
$$

Proof We first give the lower estimate for the essential norm. Without loss of generality, we assume that $n \geq m+1$. Choose the sequence of function $f_{n}(z)=z^{n} /\left\|z^{n}\right\|_{\mathcal{L B}}, n \in \mathbb{N}$. Then $\left\|f_{n}\right\|_{\mathcal{L B}}=1$, and $\left\{f_{n}\right\}$ converges to zero weakly on $\mathcal{L B}$ as $n \rightarrow \infty$. Thus we have

$$
\lim _{n \rightarrow \infty}\left\|K f_{n}\right\|_{\mathcal{L B}}=0
$$


for any given compact operator $K$ on $\mathcal{L B}$. The basic inequality gives

$$
\left\|C_{\varphi} D^{m}-K\right\|^{\mathcal{L B} \rightarrow \mathcal{L B}} \geq\left\|\left(C_{\varphi} D^{m}-K\right) f_{n}\right\|_{\mathcal{L B}} \geq\left\|C_{\varphi} D^{m} f_{n}\right\|_{\mathcal{L B}}-\left\|K f_{n}\right\|_{\mathcal{L B}}
$$

Thus we obtain

$$
\left\|C_{\varphi} D^{m}-K\right\|^{\mathcal{L B} \rightarrow \mathcal{L B}} \geq \limsup _{n \rightarrow \infty}\left\|C_{\varphi} D^{m} f_{n}\right\|_{\mathcal{L B}} \geq \limsup _{n \rightarrow \infty}\left\|C_{\varphi} D^{m} f_{n}\right\|_{\mathcal{L B}}
$$

So we have

$$
\left\|C_{\varphi} D^{m}\right\|_{e}^{\mathcal{L B} \rightarrow \mathcal{L B}}=\inf _{K}\left\|C_{\varphi} D^{m}-K\right\| \geq \limsup _{n \rightarrow \infty} \frac{\left\|C_{\varphi} D^{m}\left(z^{n}\right)\right\|_{\mathcal{L B}}}{\left\|z^{n}\right\|_{\mathcal{L B}}} .
$$

Now we give the upper estimate for the essential norm. For the case of $\sup _{z \in \mathbb{D}}|\varphi(z)|<1$, there is a number $\delta \in(0,1)$ such that $\sup _{z \in \mathbb{D}}|\varphi(z)|<\delta$. In this case, the operator $C_{\varphi} D^{m}$ is compact on $\mathcal{L B}$. In fact, choose a bounded sequence $\left\{f_{n}\right\}_{n \in \mathbb{N}}$ in $\mathcal{L B}$ which converges to zero uniformly on compact subset of $\mathbb{D}$. From Cauchy's integral formula, $\left\{f_{n}^{(m+1)}\right\}$ converges to zero on compact subsets of $\mathbb{D}$ as $n \rightarrow \infty$. It follows that

$$
\begin{aligned}
\lim _{n \rightarrow \infty}\left\|C_{\varphi} D^{m} f_{n}\right\|_{\mathcal{L B}} & =\lim _{n \rightarrow \infty}\left(\left|f_{n}^{(m)}(\varphi(0))\right|+\left\|C_{\varphi} D^{m} f_{n}\right\|_{\text {log }}\right) \\
& =\lim _{n \rightarrow \infty} \sup _{z \in \mathbb{D}}(1-|z|) \log \frac{e}{1-|z|}\left|f_{n}^{(m+1)}(\varphi(z)) \varphi^{\prime}(z)\right| \\
& \leq\|\varphi\|_{\mathcal{L B}} \lim _{n \rightarrow \infty} \sup _{z \in \mathbb{D}}\left|f_{n}^{(m+1)}(\varphi(z))\right| \\
& =\|\varphi\|_{\mathcal{L B}} \lim _{n \rightarrow \infty} \sup _{|w| \leq \delta}\left|f_{n}^{(m+1)}(w)\right|=0 .
\end{aligned}
$$

Then the operator $C_{\varphi} D^{m}$ is compact on $\mathcal{L B}$ by Lemma 4.2. This gives

$$
\left\|C_{\varphi} D^{m}\right\|_{e}^{\mathcal{L B} \rightarrow \mathcal{L B}}=0
$$

On the other hand, by Lemma 2.1 and (2.9) we obtain

$$
\left\|z^{n}\right\|_{\mathcal{L B}}=H_{0, n}\left(x_{0, n}\right) \geq H_{0, n}\left(r_{0, n}\right) \geq \frac{1}{2} \log (e n)
$$

which implies that

$$
\begin{aligned}
& \limsup _{n \rightarrow \infty} \frac{\left\|C_{\varphi} D^{m}\left(z^{n}\right)\right\|_{\mathcal{L B}}}{\left\|z^{n}\right\|_{\mathcal{L B}}} \\
& \quad \leq e \limsup _{n \rightarrow \infty} \sup _{z \in \mathbb{D}}(1-|z|) \log \frac{e}{1-|z|} \frac{n !}{(n-m-1) !}|\varphi(z)|^{n-m-1}\left|\varphi^{\prime}(z)\right| \\
& \quad \leq e\|\varphi\|_{\mathcal{L B}} \lim _{n \rightarrow \infty} n^{m} \delta^{n-m-1}=0 .
\end{aligned}
$$

Combining the last inequality with (4.3), we get the desired result.

Next, we assume that $\sup _{z \in \mathbb{D}}|\varphi(z)|=1$. Let $L_{n}$ be the sequence of operators given in Lemma 4.1. Since $L_{n}$ is compact on $\mathcal{L B}$ and $C_{\varphi} D^{m}$ is bounded on $\mathcal{L B}$, then $C_{\varphi} D^{m} L_{n}$ is also 
compact on $\mathcal{L B}$. Hence

$$
\begin{aligned}
\left\|C_{\varphi} D^{m}\right\|_{e}^{\mathcal{L B} \rightarrow \mathcal{L B}} & \leq \limsup _{n \rightarrow \infty}\left\|C_{\varphi} D^{m}-C_{\varphi} D^{m} L_{n}\right\|_{\mathcal{L B} \rightarrow \mathcal{L B}} \\
& =\limsup _{n \rightarrow \infty}\left\|C_{\varphi} D^{m}\left(I-L_{n}\right)\right\|_{\mathcal{L B} \rightarrow \mathcal{L B}} \\
& =\limsup _{n \rightarrow \infty} \sup _{\|f\|_{\mathcal{L B}} \leq 1}\left\|C_{\varphi} D^{m}\left(I-L_{n}\right) f\right\|_{\mathcal{L B}} \\
& =\limsup _{n \rightarrow \infty} \sup _{\|f\|_{\mathcal{L B}} \leq 1}\left\|\left(\left(I-L_{n}\right) f\right)^{(m)} \circ \varphi\right\|_{\mathcal{L B}} \\
& \leq I_{1}+I_{2},
\end{aligned}
$$

where

$$
I_{1}=\limsup _{n \rightarrow \infty} \sup _{\|f\|_{\mathcal{L B}} \leq 1}\left|\left(\left(I-L_{n}\right) f\right)^{(m)}(\varphi(0))\right|
$$

and

$$
I_{2}=\limsup _{n \rightarrow \infty} \sup _{\|f\|_{\mathcal{L B}} \leq 1} \sup _{z \in \mathbb{D}}\left|\left(\left(I-L_{n}\right) f\right)^{(m+1)}(\varphi(z)) \varphi^{\prime}(z)\right|(1-|z|) \log \frac{e}{1-|z|} .
$$

It follows from Lemma 4.1(iib) and Cauchy's integral formula that $I_{1}=0$.

For each positive integer $n \geq m+1$, we define

$$
\mathbb{D}_{n}=\left\{z \in \mathbb{D}: r_{m, n-m} \leq|\varphi(z)|<r_{m, n}\right\},
$$

where $r_{m, n}$ is given in Lemma 2.1. Let $k$ be the smallest positive integer such that $\mathbb{D}_{k} \neq 0$. Since $\sup _{z \in \mathbb{D}}|\varphi(z)|=1, \mathbb{D}_{n}$ is not empty for every integer $n \geq k$ and $\mathbb{D}=\bigcup_{n=k}^{\infty} \mathbb{D}_{n}$, we have

$$
\sup _{\|f\|_{\mathcal{L B}} \leq 1} \sup _{z \in \mathbb{D}}\left|\left(\left(I-L_{n}\right) f\right)^{(m+1)}(\varphi(z)) \varphi^{\prime}(z)\right|(1-|z|) \log \frac{e}{1-|z|}=I_{21}+I_{22},
$$

where

$$
I_{21}=\sup _{\|f\|_{\mathcal{L} \mathcal{B}} \leq 1} \sup _{k \leq i \leq N-1} \sup _{z \in \mathbb{D}_{i}}\left|\left(\left(I-L_{n}\right) f\right)^{(m+1)}(\varphi(z)) \varphi^{\prime}(z)\right|(1-|z|) \log \frac{e}{1-|z|}
$$

and

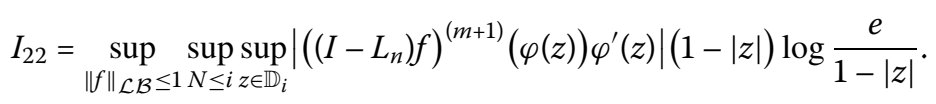

Here $N$ is a positive integer determined as follows.

By (2.8),

$$
\lim _{i \rightarrow \infty} \frac{\left\|z^{i}\right\|_{\mathcal{L B}}}{H_{m, i}\left(r_{m, i-m}\right)}=\frac{e^{m}}{(m+1)^{m+1}} .
$$

Hence, for any given $\varepsilon>0$, there exists an $N$ such that

$$
\frac{\left\|z^{i}\right\|_{\mathcal{L B}}}{H_{m, i}\left(r_{m, i-m}\right)} \leq \frac{e^{m}}{(m+1)^{m+1}}+\varepsilon
$$


when $i \geq N$. For such $N$ it follows that

$$
\begin{aligned}
& I_{22}=\sup _{\|f\|_{\mathcal{L B}} \leq 1} \sup _{N \leq i} \sup _{z \in \mathbb{D}_{i}}\left|\left(\left(I-L_{n}\right) f\right)^{(m+1)}(\varphi(z)) \varphi^{\prime}(z)\right|(1-|z|) \log \frac{e}{1-|z|} \\
& =\sup _{\|f\|_{\mathcal{L B}} \leq 1} \sup _{N \leq i} \sup _{z \in \mathbb{D}_{i}}\left|\left(\left(I-L_{n}\right) f\right)^{(m+1)}(\varphi(z)) \varphi^{\prime}(z)\right| \\
& \cdot(1-|z|) \log \frac{e}{1-|z|} \frac{H_{m, i}(|\varphi(z)|)}{\left\|z^{i}\right\|_{\mathcal{L B}}} \frac{\left\|z^{i}\right\|_{\mathcal{L B}}}{H_{m, i}(|\varphi(z)|)} \\
& \preceq\left(\frac{e^{m}}{(m+1)^{m+1}}+\varepsilon\right) \sup _{\|f\|_{\mathcal{L B}} \leq 1}\left\|\left(I-L_{n}\right) f\right\|_{\mathcal{L B}} \sup _{N \leq i} \sup _{z \in \mathbb{D}_{i}}\left|\varphi^{\prime}(z)\right| \\
& \cdot(1-|z|) \log \frac{e}{1-|z|} \frac{i !}{(i-m-1) !} \frac{|\varphi(z)|^{i-m-1}}{\left\|z^{i}\right\|_{\mathcal{L B}}} \\
& \leq\left(\frac{e^{m}}{(m+1)^{m+1}}+\varepsilon\right)\left\|I-L_{n}\right\| \sup _{N \leq i} \frac{\left\|C_{\varphi} D^{m}\left(z^{i}\right)\right\|_{\mathcal{L B}}}{\left\|z^{i}\right\|_{\mathcal{L B}}}
\end{aligned}
$$

Thus

$$
\limsup _{n \rightarrow \infty} I_{22} \preceq\left(\frac{e^{m}}{(m+1)^{m+1}}+\varepsilon\right) \sup _{N \leq i} \frac{\left\|C_{\varphi} D^{m}\left(z^{i}\right)\right\|_{\mathcal{L B}}}{\left\|z^{i}\right\|_{\mathcal{L B}}} .
$$

By (ii) of Lemma 4.1 and Cauchy's integral formula, we have

$$
\begin{aligned}
& \underset{n \rightarrow \infty}{\lim \sup } I_{21} \\
& \quad=\limsup _{n \rightarrow \infty} \sup _{\|f\|_{\mathcal{L B}} \leq 1} \sup _{k \leq i<N-1} \sup _{z \in \mathbb{D}_{i}}\left|\left(\left(I-L_{n}\right) f\right)^{(m+1)}(\varphi(z))\right|\left|\varphi^{\prime}(z)\right|(1-|z|) \log \frac{e}{1-|z|} \\
& \quad \leq\|\varphi\|_{\mathcal{L B}} \limsup _{n \rightarrow \infty} \sup _{\|f\|_{\mathcal{L} \mathcal{B} \leq 1}} \sup _{|\varphi(z)|<r_{m, N-1}}\left|\left(\left(I-L_{n}\right) f\right)^{(m+1)}(\varphi(z))\right| \\
& \quad=0
\end{aligned}
$$

which together with (4.4) implies that

$$
I_{2} \preceq\left(\frac{e^{m}}{(m+1)^{m+1}}+\varepsilon\right) \sup _{N \leq i} \frac{\left\|C_{\varphi} D^{m}\left(z^{i}\right)\right\|_{\mathcal{L B}}}{\left\|z^{i}\right\|_{\mathcal{L B}}} .
$$

From (4.5) we obtain

$$
\left\|C_{\varphi} D^{m}\right\|_{e}^{\mathcal{L B} \rightarrow \mathcal{L B}} \leq I_{1}+I_{2} \preceq\left(\frac{e^{m}}{(m+1)^{m+1}}+\varepsilon\right) \sup _{N \leq i} \frac{\left\|C_{\varphi} D^{m}\left(z^{i}\right)\right\|_{\mathcal{L B}}}{\left\|z^{i}\right\|_{\mathcal{L B}}} .
$$

By the arbitrariness of $\varepsilon$, we get

$$
\left\|C_{\varphi} D^{m}\right\|_{e}^{\mathcal{L B} \rightarrow \mathcal{L B}} \preceq \frac{e^{m}}{(m+1)^{m+1}} \limsup _{n \rightarrow \infty} \frac{\left\|C_{\varphi} D^{m}\left(z^{n}\right)\right\|_{\mathcal{L B}}}{\left\|z^{n}\right\|_{\mathcal{L B}}} .
$$

The proof is complete.

From Theorem 4.3, we obtain the following result. 
Corollary 4.4 Let $m \in \mathbb{N}$ and $\varphi$ be an analytic self-map of $\mathbb{D}$ such that $C_{\varphi} D^{m}$ is bounded on $\mathcal{L B}$. Then $C_{\varphi} D^{m}$ is compact on $\mathcal{L B}$ if and only if

$$
\limsup _{n \rightarrow \infty} \frac{\left\|C_{\varphi} D^{m}\left(z^{n}\right)\right\|_{\mathcal{L B}}}{\left\|z^{n}\right\|_{\mathcal{L B}}}=0
$$

Especially, when $m=0$, from the proof of Theorem 4.3, we get the exact formula for essential norm of composition operator on $\mathcal{L B}$.

Corollary 4.5 Let $\varphi$ be an analytic self-map of $\mathbb{D}$. Suppose that $C_{\varphi}$ is bounded on $\mathcal{L B}$; then

$$
\left\|C_{\varphi}\right\|_{e}^{\mathcal{L B} \rightarrow \mathcal{L B}}=\limsup _{n \rightarrow \infty} \frac{\left\|\varphi^{n}\right\|_{\mathcal{L B}}}{\left\|z^{n}\right\|_{\mathcal{L B}}}
$$

\section{Competing interests}

The authors declare that they have no competing interests.

\section{Authors' contributions}

All authors contributed equally to the writing of this paper. All authors read and approved the final manuscript.

\section{Author details}

${ }^{1}$ School of Sciences, Anhui University of Science and Technology, Huainan, Anhui 232001, China. ${ }^{2}$ Faculty of Information Technology, Macau University of Science and Technology, Avenida Wai Long, Taipa, Macau, China.

\section{Acknowledgements}

The project is supported by Department of education of Anhui Province of China (No. KJ2013A101), the Natural Science Foundation of Guangdong (No. S2013010011978) and NSF of China (No. 11471143).

Received: 22 September 2014 Accepted: 29 October 2014 Published: 10 Nov 2014

\section{References}

1. Brown, L, Shields, A: Multipliers and cyclic vectors in the Bloch space. Mich. Math. J. 38, 141-146 (1991)

2. Zhu, K: Operator Theory in Function Spaces. Dekker, New York (1990)

3. Han, J, Wu, Y: The high order derivative characterization of logarithmic Bloch type spaces. J. Anhui Univ. Sci. Technol. 2, 32-34 (2013)

4. Krantz, S, Stević, S: On the iterated logarithmic Bloch space on the unit ball. Nonlinear Anal. TMA 71, 1772-1795 (2009)

5. Stević, S: Generalized composition operators from logarithmic Bloch spaces to mixed-norm spaces. Util. Math. 77 167-172 (2008)

6. Stević, S: On a new operator from the logarithmic Bloch space to the Bloch-type space on the unit ball. Appl. Math. Comput. 206, 313-320 (2008)

7. Stević, S: On an integral-type operator from logarithmic Bloch-type and mixed-norm spaces to Bloch-type spaces. Nonlinear Anal. TMA 71, 6323-6342 (2009)

8. Stević, S: Norm of some operators from logarithmic Bloch-type spaces to weighted-type spaces. Appl. Math. Comput. 218, 1163-1170 (2012)

9. Stević, S, Agarwal, R: Weighted composition operators from logarithmic Bloch-type spaces to Bloch-type spaces. J. Inequal. Appl. 2009, Article ID 964814 (2009)

10. Yoneda, R: The composition operators on weighted Bloch space. Arch. Math. (Basel) 78, 310-317 (2002)

11. Cowen, C, Maccluer, B: Composition Operators on Spaces of Analytic Functions. CRC Press, Boca Raton (1995)

12. Hibschweiler, R, Portnoy, N: Composition followed by differentiation between Bergman and Hardy spaces. Rocky Mt. J. Math. 35, 843-855 (2005)

13. Li, S, Stević, S: Composition followed by differentiation between Bloch type spaces. J. Comput. Anal. Appl. 9, 195-205 (2007)

14. Li, S, Stević, S: Weighted composition operators from Bergman-type spaces into Bloch spaces. Proc. Indian Acad. Sci. Math. Sci. 117, 371-385 (2007)

15. Li, S, Stević, S: Weighted composition operators from $H^{\infty}$ to the Bloch space on the polydisc. Abstr. Appl. Anal. 2007, Article ID 48478 (2007)

16. Li, S, Stević, S: Products of composition and integral type operators from $H^{\infty}$ to the Bloch space. Complex Var. Elliptic Equ. 53(5), 463-474 (2008)

17. Li, S, Stević, S: Weighted composition operators from Zygmund spaces into Bloch spaces. Appl. Math. Comput. 206(2), 825-831 (2008)

18. Li, S, Stević, S: Composition followed by differentiation between $H^{\infty}$ and $\alpha$-Bloch spaces. Houst. J. Math. 35, 327-340 (2009) 
19. Li, S, Stević, S: Products of integral-type operators and composition operators between Bloch-type spaces. J. Math. Anal. Appl. 349, 596-610 (2009)

20. Liang, Y, Zhou, Z: Essential norm of the product of differentiation and composition operators between Bloch-type space. Arch. Math. 100, 347-360 (2013)

21. Lou, Z: Composition operators on Bloch type spaces. Analysis 23, $81-95$ (2003)

22. Maccluer, B, Zhao, R: Essential norms of weighted composition operators between Bloch-type spaces. Rocky Mt. J. Math. 33, 1437-1458 (2003)

23. Madigan, K, Matheson, A: Compact composition operators on the Bloch space. Trans. Am. Math. Soc. 347, 2679-2687 (1995)

24. Montes-Rodriguez, A: The essential norm of a composition operator on Bloch spaces. Pac. J. Math. 188, 339-351 (1999)

25. Montes-Rodriguez, A: Weighted composition operators on weighted Banach spaces of analytic functions. J. Lond. Math. Soc. 61, 872-884 (2000)

26. Stević, S: Essential norms of weighted composition operators from the $\alpha$-Bloch space to a weighted-type space on the unit ball. Abstr. Appl. Anal. 2008, Article ID 279691 (2008)

27. Stević, S: Norms of some operators from Bergman spaces to weighted and Bloch-type space. Util. Math. 76, 59-64 (2008)

28. Stević, S: Norm and essential norm of composition followed by differentiation from $\alpha$-Bloch spaces to $H_{\mu}^{\infty}$. Appl. Math. Comput. 207, 225-229 (2009)

29. Stević, S: On a new integral-type operator from the Bloch space to Bloch-type spaces on the unit ball. J. Math. Anal. Appl. 354, 426-434 (2009)

30. Stević, S: Products of integral-type operators and composition operators from the mixed norm space to Bloch-type spaces. Sib. Math. J. 50(4), 726-736 (2009)

31. Stević, S: On an integral operator between Bloch-type spaces on the unit ball. Bull. Sci. Math. 134, $329-339$ (2010)

32. Stević, S: Weighted differentiation composition operators from $H^{\infty}$ and Bloch spaces to $n$th weigthed-type spaces on the unit disk. Appl. Math. Comput. 216, 3634-3641 (2010)

33. Tjani, M: Compact composition operators on some Möbius invariant Banach space. PhD dissertation, Michigan State University (1996)

34. Wu, Y, Wulan, H: Products of differentiation and composition operators on the Bloch space. Collect. Math. 63, 93-107 (2012)

35. Wulan, H, Zheng, D, Zhu, K: Compact composition operators on BMOA and the Bloch space. Proc. Am. Math. Soc. 137, 3861-3868 (2009)

36. Yang, W: Products of composition and differentiation operators from $Q_{K}(p, q)$ spaces to Bloch-type spaces. Abstr. Appl. Anal. 2009, Article ID 741920 (2009)

37. Zhao, R: Essential norms of composition operators between Bloch type spaces. Proc. Am. Math. Soc. 138, 2537-2546 (2010)

10.1186/1029-242X-2014-453

Cite this article as: Zhou and Zhu: Product of differentiation and composition operators on the logarithmic Bloch space. Journal of Inequalities and Applications 2014, 2014:453

\section{Submit your manuscript to a SpringerOpen ${ }^{\circ}$ journal and benefit from:}

- Convenient online submission

Rigorous peer review

- Immediate publication on acceptance

- Open access: articles freely available online

- High visibility within the field

- Retaining the copyright to your article 